Proceedings of the Institute of Mathematics and Mechanics,

National Academy of Sciences of Azerbaijan

Volume 45, Number 2, 2019, Pages 181-191

https://doi.org/10.29228/proc.2

\title{
POSITIVITY FOR INTEGRAL BOUNDARY VALUE PROBLEMS OF HADAMARD FRACTIONAL DIFFERENTIAL EQUATIONS
}

\author{
ABDELOUAHEB ARDJOUNI
}

\begin{abstract}
In this paper, we prove the existence and uniqueness of a positive solution of integral boundary value problems of nonlinear Hadamard fractional differential equations. In the process we employ the Schauder and Banach fixed point theorems and the method of upper and lower solutions to show the existence and uniqueness of a positive solution. Finally, an example is given to illustrate our results.
\end{abstract}

\section{Introduction}

Fractional differential equations arise from a variety of applications including in various fields of science and engineering. In particular, problems concerning qualitative analysis of the positivity of such solutions for fractional differential equations (FDE) have received the attention of many authors, see [1]-[7], [9], [10], [14]-[18] and the references therein.

Recently, Zhang in [18] investigated the existence and uniqueness of positive solutions for the nonlinear fractional differential equation

$$
\left\{\begin{array}{l}
D^{\alpha} x(t)=f(t, x(t)), 0<t \leq 1 \\
x(0)=0
\end{array}\right.
$$

where $D^{\alpha}$ is the standard Riemann Liouville fractional derivative of order $0<$ $\alpha<1$, and $f:[0,1] \times[0, \infty) \rightarrow[0, \infty)$ is a given continuous function. By using the method of the upper and lower solution and cone fixed-point theorem, the author obtained the existence and uniqueness of a positive solution.

The nonlinear fractional differential equation boundary value problem

$$
\left\{\begin{array}{l}
D^{\alpha} x(t)+f(t, x(t))=0,0<t<1, \\
x(0)=x(1)=0
\end{array}\right.
$$

has been investigated in [2], where $1<\alpha \leq 2$, and $f:[0,1] \times[0, \infty) \rightarrow[0, \infty)$ is a given continuous function. By means of some fixed-point theorems on cone, some existence and multiplicity results of positive solutions have been established.

2010 Mathematics Subject Classification. 26A33, 34A12, 34G20.

Key words and phrases. Fractional differential equations, positive solutions, upper and lower solutions, existence, uniqueness, fixed point theorems. 
In [10], Matar discussed the existence and uniqueness of the positive solution of the following nonlinear fractional differential equation

$$
\left\{\begin{array}{l}
{ }^{C} D^{\alpha} x(t)=f(t, x(t)), 0<t \leq 1 \\
x(0)=0, x^{\prime}(0)=\theta>0
\end{array}\right.
$$

where ${ }^{C} D^{\alpha}$ is the standard Caputo's fractional derivative of order $1<\alpha \leq 2$, and $f:[0,1] \times[0, \infty) \rightarrow[0, \infty)$ is a given continuous function. By employing the method of the upper and lower solutions and Schauder and Banach fixed point theorems, the author obtained positivity results.

The integral boundary value problems of the nonlinear fractional differential equation

$$
\left\{\begin{array}{l}
D^{\alpha} x(t)+f(t, x(t))=D^{\alpha-1} g(t, x(t)), 0<t<1, \\
x(0)=0, x(1)=\int_{0}^{1} g(s, x(s)) d s
\end{array}\right.
$$

has been investigated in [16], where $1<\alpha \leq 2, g, f:[0,1] \times[0, \infty) \rightarrow[0, \infty)$ are given continuous functions, $g$ is non-decreasing on $x$. By employing the method of the upper and lower solutions and the Schauder and Banach fixed point theorems, the authors obtained positivity results.

Benchohra and Lazreg in [4] studied the existence and uniqueness of solutions to the following boundary value problem

$$
\left\{\begin{array}{l}
\mathfrak{D}_{1}^{\alpha} x(t)=f\left(t, x(t), \mathfrak{D}_{1}^{\alpha} x(t)\right), t \in[1, T], \\
x(1)=x_{0}
\end{array}\right.
$$

where $\mathfrak{D}_{1}^{\alpha}$ is the Hadamard fractional derivatives of order $0<\alpha \leq 1$. By employing the fixed point theorems, the authors obtained existence and uniqueness results.

In this paper, we are interested in the analysis of qualitative theory of the problems of the positive solutions to fractional differential equations. Inspired and motivated by the works mentioned above and the papers [1]-[7], $[9,10]$, [14]-[18] and the references therein, we concentrate on the positivity of solutions for the integral boundary value problems of the nonlinear Hadamard fractional differential equation

$$
\left\{\begin{array}{l}
\mathfrak{D}_{1}^{\alpha} x(t)+f(t, x(t))=\mathfrak{D}_{1}^{\alpha-1} g(t, x(t)), 1<t<e \\
x(1)=0, x(e)=\theta+\int_{1}^{e} g(s, x(s)) \frac{d s}{s}
\end{array}\right.
$$

where $1<\alpha \leq 2, \theta \in[0, \infty), g, f:[1, e] \times[0, \infty) \rightarrow[0, \infty)$ are given continuous functions, $g$ is non-decreasing on $x$ and $f$ is not required any monotone assumption. To show the existence and uniqueness of the positive solution, we transform (1.1) into an integral equation and then by the method of upper and lower solutions and use the Schauder and Banach fixed point theorems.

This paper is organized as follows. In section 2, we introduce some notations and lemmas, and state some preliminaries results needed in later section. Also, we present the inversion of (1.1) and the Banach and Schauder fixed point theorems. For details on the Banach and Schauder theorems we refer the reader to [13]. In Section 3 , we give and prove our main results on positivity, and we provide an example to illustrate our results. 


\section{Preliminaries}

Let $X=C([1, e])$ be the Banach space of all real-valued continuous functions defined on the compact interval $[1, e]$, endowed with the maximum norm. Define the subspace $\mathcal{A}=\{x \in X: x(t) \geq 0, t \in[1, e]\}$ of $X$. By a positive solution $x \in X$, we mean a function $x(t)>0,1 \leq t \leq e$.

Let $a, b \in \mathbb{R}^{+}$such that $b>a$. For any $x \in[a, b]$, we define the upper-control function

and lower-control function

$$
U(t, x)=\sup \{f(t, \lambda): a \leq \lambda \leq x\},
$$

$$
L(t, x)=\inf \{f(t, \lambda): x \leq \lambda \leq b\} .
$$

Obviously, $U(t, x)$ and $L(t, x)$ are monotonous non-decreasing on the argument $x$ and $L(t, x) \leq f(t, x) \leq U(t, x)$.

We introduce some necessary definitions, lemmas and theorems which will be used in this paper. For more details, see [8, 11, 12].

Definition 2.1 ([8]). The Hadamard fractional integral of order $\alpha>0$ for a continuous function $x:[1,+\infty) \rightarrow \mathbb{R}$ is defined as

$$
\mathfrak{I}_{1}^{\alpha} x(t)=\frac{1}{\Gamma(\alpha)} \int_{1}^{t}\left(\log \frac{t}{s}\right)^{\alpha-1} x(s) \frac{d s}{s}, \alpha>0 .
$$

Definition $2.2([8])$. The Hadamard fractional derivative of order $\alpha>0$ for a continuous function $x:[1,+\infty) \rightarrow \mathbb{R}$ is defined as

$$
\mathfrak{D}_{1}^{\alpha} x(t)=\frac{1}{\Gamma(n-\alpha)}\left(t \frac{d}{d t}\right)^{n} \int_{1}^{t}\left(\log \frac{t}{s}\right)^{n-\alpha-1} x(s) \frac{d s}{s}, n-1<\alpha<n .
$$

Lemma 2.1 ([8]). Let $n-1<\alpha \leq n, n \in \mathbb{N}$. The equality $\left(\mathfrak{I}_{1}^{\alpha} \mathfrak{D}_{1}^{\alpha} x\right)(t)=0$ is valid if and only if

$$
x(t)=\sum_{k=1}^{n} c_{k}(\log t)^{\alpha-k} \text { for each } t \in[1, e],
$$

where $c_{k} \in \mathbb{R}, k=1, \ldots, n$ are arbitrary constants.

Lemma $2.2([8])$. For all $\mu>0$ and $\nu>-1$,

$$
\frac{1}{\Gamma(\mu)} \int_{1}^{t}\left(\log \frac{t}{s}\right)^{\mu-1}(\log s)^{\nu} \frac{d s}{s}=\frac{\Gamma(\nu+1)}{\Gamma(\mu+\nu+1)}(\log t)^{\mu+\nu} .
$$

The following lemma is fundamental to our results.

Lemma 2.3. Let $x \in C^{1}([1, e]), x^{(2)}$ and $\frac{\partial g}{\partial t}$ exist, then $x$ is a solution of (1.1) if and only if

$$
x(t)=\theta(\log t)^{\alpha-1}+\int_{1}^{e} H(t, s) f(s, x(s)) \frac{d s}{s}+\int_{1}^{t} g(s, x(s)) \frac{d s}{s},
$$

where

$$
H(t, s)=\left\{\begin{array}{l}
\frac{\left(\log t \log \frac{e}{s}\right)^{\alpha-1}-\left(\log \frac{t}{s}\right)^{\alpha-1}}{\Gamma(\alpha)}, 1 \leq s \leq t \leq e \\
\frac{\left(\log t \log \frac{e}{s}\right)^{\alpha-1}}{\Gamma(\alpha)}, 1 \leq t \leq s \leq e
\end{array}\right.
$$


Proof. Let $x$ be a solution of (1.1). First we write this equation as

$$
\mathfrak{I}_{1}^{\alpha} \mathfrak{D}_{1}^{\alpha} x(t)=\mathfrak{I}_{1}^{\alpha}\left(-f(t, x(t))+\mathfrak{D}_{1}^{\alpha-1} g(t, x(t))\right), 1<t<e .
$$

From Lemma 2.1, we have

$$
\begin{aligned}
& x(t)-c_{2}(\log t)^{\alpha-2}-c_{1}(\log t)^{\alpha-1} \\
& =-\mathfrak{I}_{1}^{\alpha} f(t, x(t))+\mathfrak{I}_{1}^{\alpha} \mathfrak{D}_{1}^{\alpha-1} g(t, x(t)) \\
& =-\mathfrak{I}_{1}^{\alpha} f(t, x(t))+\mathfrak{I}_{1}^{1} \mathfrak{I}_{1}^{\alpha-1} \mathfrak{D}_{1}^{\alpha-1} g(t, x(t)) \\
& =-\mathfrak{I}_{1}^{\alpha} f(t, x(t))+\mathfrak{I}_{1}^{1}\left(g(t, x(t))-c_{3}(\log t)^{\alpha-2}\right) \\
& =-\frac{1}{\Gamma(\alpha)} \int_{1}^{t}\left(\log \frac{t}{s}\right)^{\alpha-1} f(s, x(s)) \frac{d s}{s} \\
& +\int_{1}^{t} g(s, x(s)) \frac{d s}{s}-c_{3} \frac{(\log t)^{\alpha-1}}{\alpha-1} .
\end{aligned}
$$

Then

$$
\begin{aligned}
x(t) & =c_{2}+\left(c_{1}-\frac{c_{3}}{\alpha-1}\right)(\log t)^{\alpha-1} \\
& -\frac{1}{\Gamma(\alpha)} \int_{1}^{t}\left(\log \frac{t}{s}\right)^{\alpha-1} f(s, x(s)) \frac{d s}{s}+\int_{1}^{t} g(s, x(s)) \frac{d s}{s} .
\end{aligned}
$$

By boundary conditions $x(1)=0$ and $x(e)=\theta+\int_{1}^{e} g(s, x(s)) \frac{d s}{s}$, we obtain $c_{2}=0$ and

$$
c_{1}-\frac{c_{3}}{\alpha-1}=\theta+\frac{1}{\Gamma(\alpha)} \int_{1}^{e}\left(\log \frac{e}{s}\right)^{\alpha-1} f(s, x(s)) \frac{d s}{s} .
$$

Therefore

$$
\begin{aligned}
x(t) & =\theta(\log t)^{\alpha-1}+\frac{1}{\Gamma(\alpha)} \int_{1}^{e}\left(\log t \log \frac{e}{s}\right)^{\alpha-1} f(s, x(s)) \frac{d s}{s} \\
& -\frac{1}{\Gamma(\alpha)} \int_{1}^{t}\left(\log \frac{t}{s}\right)^{\alpha-1} f(s, x(s)) \frac{d s}{s}+\int_{1}^{t} g(s, x(s)) \frac{d s}{s} \\
& =\theta(\log t)^{\alpha-1}+\int_{1}^{e} H(t, s) f(s, x(s)) \frac{d s}{s}+\int_{1}^{t} g(s, x(s)) \frac{d s}{s} .
\end{aligned}
$$

Since each step is reversible, the converse follows easily. This completes the proof.

Corollary 2.1. The function $H$ defined by (2.2) satisfies

(1) $H(t, s)>0$ for $t, s \in(1, e)$,

(2) $\max _{1 \leq t \leq e} H(t, s)=H(s, s), s \in(1, e)$.

Lastly in this section, we state the fixed point theorems which enable us to prove the existence and uniqueness of a positive solution of (1.1).

Definition 2.3. Let $(X,\|\|$.$) be a Banach space and \Phi: X \rightarrow X$. The operator $\Phi$ is a contraction operator if there is an $\lambda \in(0,1)$ such that $x, y \in X$ imply

$$
\|\Phi x-\Phi y\| \leq \lambda\|x-y\| \text {. }
$$


Theorem 2.1 (Banach [13]). Let $\mathcal{C}$ be a nonempty closed convex subset of a Banach space $X$ and $\Phi: \mathcal{C} \rightarrow \mathcal{C}$ be a contraction operator. Then there is a unique $x \in \mathcal{C}$ with $\Phi x=x$.

Theorem 2.2 (Schauder [13]). Let $\mathcal{C}$ be a nonempty closed convex subset of a Banach space $X$ and $\Phi: \mathcal{C} \rightarrow \mathcal{C}$ be a continuous compact operator. Then $\Phi$ has a fixed point in $\mathcal{C}$.

\section{Main results}

In this section, we consider the results of existence problem for many cases of the FDE (1.1). Moreover, we introduce the sufficient conditions of the uniqueness problem of (1.1).

To transform equation (2.1) to be applicable to Schauder fixed point, we define an operator $\Phi: \mathcal{A} \longrightarrow X$ by

$$
(\Phi x)(t)=\theta(\log t)^{\alpha-1}+\int_{1}^{e} H(t, s) f(s, x(s)) \frac{d s}{s}+\int_{1}^{t} g(s, x(s)) \frac{d s}{s}, t \in[1, e],
$$

where the figured fixed point must satisfy the identity operator equation $\Phi x=x$.

The following assumptions are needed for the next results.

(H1) Let $x^{*}, x_{*} \in \mathcal{A}$, such that $a \leq x_{*}(t) \leq x^{*}(t) \leq b$ and

$$
\left\{\begin{array}{l}
\mathfrak{D}_{1}^{\alpha} x^{*}(t)+U\left(t, x^{*}(t)\right) \geq \mathfrak{D}_{1}^{\alpha-1} g\left(t, x^{*}(t)\right) \\
\mathfrak{D}_{1}^{\alpha} x_{*}(t)+L\left(t, x_{*}(t)\right) \leq \mathfrak{D}_{1}^{\alpha-1} g\left(t, x_{*}(t)\right)
\end{array}\right.
$$

for any $t \in[1, e]$.

$(H 2)$ For $t \in[1, e]$ and $x, y \in X$, there exist positive real numbers $\beta_{1}, \beta_{2}<1$ such that

$$
\begin{aligned}
|g(t, y)-g(t, x)| & \leq \beta_{1}\|y-x\|, \\
|f(t, y)-f(t, x)| & \leq \beta_{2}\|y-x\| .
\end{aligned}
$$

The functions $x^{*}$ and $x_{*}$ are respectively called the pair of upper and lower solutions for Equation (1.1).

Theorem 3.1. Assume that $(H 1)$ is satisfied, then the FDE (1.1) has at least one solution $x \in X$ satisfying $x_{*}(t) \leq x(t) \leq x^{*}(t), t \in[1, e]$.

Proof. Let $\mathcal{C}=\left\{x \in \mathcal{A}: x_{*}(t) \leq x(t) \leq x^{*}(t), t \in[1, e]\right\}$, endowed with the norm $\|x\|=\max _{t \in[1, e]}|x(t)|$, then we have $\|x\| \leq b$. Hence, $\mathcal{C}$ is a convex, bounded, and closed subset of the Banach space $X$. Moreover, the continuity of $g$ and $f$ implies the continuity of the operator $\Phi$ on $\mathcal{C}$ defined by (3.1). Now, if $x \in \mathcal{C}$, there exist positive constants $c_{f}$ and $c_{g}$ such that

$$
\max \{f(t, x(t)): t \in[1, e], x(t) \leq b\}<c_{f},
$$

and

$$
\max \{g(t, x(t)): t \in[1, e], x(t) \leq b\}<c_{g} .
$$


Then

$$
\begin{aligned}
|(\Phi x)(t)| & \leq \theta(\log t)^{\alpha-1}+\int_{1}^{e} H(s, s)|f(s, x(s))| \frac{d s}{s}+\int_{1}^{t}|g(s, x(s))| \frac{d s}{s} \\
& \leq \theta+\int_{1}^{e} \frac{\left(\log s \log \frac{e}{s}\right)^{\alpha-1}}{\Gamma(\alpha)} c_{f} \frac{d s}{s}+c_{g} \log t \\
& \leq \theta+\frac{\Gamma(\alpha) c_{f}}{\Gamma(2 \alpha)}+c_{g} .
\end{aligned}
$$

Thus,

$$
\|\Phi x\| \leq \theta+\frac{\Gamma(\alpha) c_{f}}{\Gamma(2 \alpha)}+c_{g} .
$$

Hence, $\Phi(\mathcal{C})$ is uniformly bounded. Next, we prove the equicontinuity of $\Phi(\mathcal{C})$. Let $x \in \mathcal{C}$, then for any $t_{1}, t_{2} \in[1, e], t_{2}>t_{1}$, we have

$$
\begin{aligned}
& \left|(\Phi x)\left(t_{2}\right)-(\Phi x)\left(t_{1}\right)\right| \\
& \leq \theta\left(\log t_{2}\right)^{\alpha-1}-\theta\left(\log t_{1}\right)^{\alpha-1} \\
& +\frac{1}{\Gamma(\alpha)}\left|\int_{1}^{e}\left(\log t_{2} \log \frac{e}{s}\right)^{\alpha-1} f(s, x(s)) \frac{d s}{s}-\int_{1}^{e}\left(\log t_{1} \log \frac{e}{s}\right)^{\alpha-1} f(s, x(s)) \frac{d s}{s}\right| \\
& +\frac{1}{\Gamma(\alpha)}\left|\int_{1}^{t_{2}}\left(\log \frac{t_{2}}{s}\right)^{\alpha-1} f(s, x(s)) \frac{d s}{s}-\int_{1}^{t_{1}}\left(\log \frac{t_{1}}{s}\right)^{\alpha-1} f(s, x(s)) \frac{d s}{s}\right| \\
& +\left|\int_{1}^{t_{2}} g(s, x(s)) \frac{d s}{s}-\int_{1}^{t_{1}} g(s, x(s)) \frac{d s}{s}\right| \\
& \leq \theta\left(\left(\log t_{2}\right)^{\alpha-1}-\left(\log t_{1}\right)^{\alpha-1}\right) \\
& +\frac{\left(\log t_{2}\right)^{\alpha-1}-\left(\log t_{1}\right)^{\alpha-1} \int_{1}^{e}\left(\log \frac{e}{s}\right)^{\alpha-1}|f(s, x(s))| \frac{d s}{s}}{\Gamma(\alpha)}-\frac{1}{\Gamma(\alpha)} \int_{1}^{t_{1}}\left(\left(\log \frac{t_{2}}{s}\right)^{\alpha-1}-\left(\log \frac{t_{1}}{s}\right)^{\alpha-1}\right)|f(s, x(s))| \frac{d s}{s} \\
& +\frac{1}{\Gamma(\alpha)} \int_{t_{1}}^{t_{2}}\left(\log \frac{t_{2}}{s}\right)^{\alpha-1}|f(s, x(s))| \frac{d s}{s}+\int_{t_{1}}^{t_{2}}|g(s, x(s))| \frac{d s}{s} \\
& \leq\left(\theta+\frac{c_{f}}{\Gamma(\alpha+1)}\right)\left(\left(\log t_{2}\right)^{\alpha-1}-\left(\log t_{1}\right)^{\alpha-1}\right) \\
& +\frac{c_{f}}{\Gamma(\alpha+1)}\left(\left(\log t_{2}\right)^{\alpha}-\left(\log t_{1}\right)^{\alpha}\right)+c_{g}\left(\log \frac{t_{2}}{t_{1}}\right) .
\end{aligned}
$$

As $t_{1} \rightarrow t_{2}$ the right-hand side of the previous inequality is independent of $x$ and tends to zero. Therefore, $\Phi(\mathcal{C})$ is equicontinuous. The Arzelè-Ascoli Theorem implies that $\Phi: \mathcal{C} \longrightarrow X$ is compact. The only thing to apply Schauder fixed 
point is to prove that $\Phi(\mathcal{C}) \subseteq \mathcal{C}$. Let $x \in \mathcal{C}$, then by hypotheses, we have

$$
\begin{aligned}
(\Phi x)(t) & =\theta(\log t)^{\alpha-1}+\int_{1}^{e} H(t, s) f(s, x(s)) \frac{d s}{s}+\int_{1}^{t} g(s, x(s)) \frac{d s}{s} \\
& \leq \theta(\log t)^{\alpha-1}+\int_{1}^{e} H(t, s) U(s, x(s)) \frac{d s}{s}+\int_{1}^{t} g\left(s, x^{*}(s)\right) \frac{d s}{s} \\
& \leq \theta(\log t)^{\alpha-1}+\int_{1}^{e} H(t, s) U\left(s, x^{*}(s)\right) \frac{d s}{s}+\int_{1}^{t} g\left(s, x^{*}(s)\right) \frac{d s}{s} \\
& \leq x^{*}(t),
\end{aligned}
$$

and

$$
\begin{aligned}
(\Phi x)(t) & =\theta(\log t)^{\alpha-1}+\int_{1}^{e} H(t, s) f(s, x(s)) \frac{d s}{s}+\int_{1}^{t} g(s, x(s)) \frac{d s}{s} \\
& \geq \theta(\log t)^{\alpha-1}+\int_{1}^{e} H(t, s) L(t, x(s)) \frac{d s}{s}+\int_{1}^{t} g\left(s, x_{*}(s)\right) \frac{d s}{s} \\
& \geq \theta(\log t)^{\alpha-1}+\int_{1}^{e} H(t, s) L\left(t, x_{*}(s)\right) \frac{d s}{s}+\int_{1}^{t} g\left(s, x_{*}(s)\right) \frac{d s}{s} \\
& \geq x_{*}(t) .
\end{aligned}
$$

Hence, $x_{*}(t) \leq(\Phi x)(t) \leq x^{*}(t), t \in[1, e]$, that is, $\Phi(\mathcal{C}) \subseteq \mathcal{C}$. According to Schauder fixed point theorem, the operator $\Phi$ has at least one fixed point $x \in \mathcal{C}$. Therefore, the FDE (1.1) has at least one positive solution $x \in X$ and $x_{*}(t) \leq$ $x(t) \leq x^{*}(t), t \in[1, e]$.

Next, we consider many particular cases of the previous theorem.

Corollary 3.1. Assume that there exist continuous functions $k_{1}, k_{2}, k_{3}$ and $k_{4}$ such that

$$
0<k_{1}(t) \leq g(t, x(t)) \leq k_{2}(t)<\infty,(t, x(t)) \in[1, e] \times[0,+\infty),
$$

and

$$
0<k_{3}(t) \leq f(t, x(t)) \leq k_{4}(t)<\infty,(t, x(t)) \in[1, e] \times[0,+\infty) .
$$

Then, the FDE (1.1) has at least one positive solution $x \in X$. Moreover,

$$
\begin{aligned}
& \theta(\log t)^{\alpha-1}+\int_{1}^{e} H(t, s) k_{3}(s) \frac{d s}{s}+\int_{1}^{t} k_{1}(s) \frac{d s}{s} \\
& \leq x(t) \\
& \leq \theta(\log t)^{\alpha-1}+\int_{1}^{e} H(t, s) k_{4}(s) \frac{d s}{s}+\int_{1}^{t} k_{2}(s) \frac{d s}{s} .
\end{aligned}
$$

Proof. By the given assumption (3.3) and the definition of control function, we have $k_{3}(t) \leq L(t, x) \leq U(t, x) \leq k_{4}(t),(t, x(t)) \in[1, e] \times[a, b]$. Now, we consider the equations

$$
\left\{\begin{array}{l}
\mathfrak{D}_{1}^{\alpha} x(t)+k_{3}(t)=\mathfrak{D}_{1}^{\alpha-1} k_{1}(t), \\
x(1)=0, x(e)=\theta+\int_{1}^{e} k_{1}(s) \frac{d s}{s}, \\
\mathfrak{D}_{1}^{\alpha} x(t)+k_{4}(t)=\mathfrak{D}_{1}^{\alpha-1} k_{2}(t) \\
x(1)=0, x(e)=\theta+\int_{1}^{e} k_{2}(s) \frac{d s}{s} .
\end{array}\right.
$$


Obviously, Equations (3.5) are equivalent to

$$
\begin{aligned}
& x(t)=\theta(\log t)^{\alpha-1}+\int_{1}^{e} H(t, s) k_{3}(s) \frac{d s}{s}+\int_{1}^{t} k_{1}(s) \frac{d s}{s}, \\
& x(t)=\theta(\log t)^{\alpha-1}+\int_{1}^{e} H(t, s) k_{4}(s) \frac{d s}{s}+\int_{1}^{t} k_{2}(s) \frac{d s}{s} .
\end{aligned}
$$

Hence, the first implies

$$
\begin{aligned}
x(t)-\int_{1}^{t} k_{1}(s) \frac{d s}{s} & =\theta(\log t)^{\alpha-1}+\int_{1}^{e} H(t, s) k_{3}(s) \frac{d s}{s} \\
& \leq \theta(\log t)^{\alpha-1}+\int_{1}^{e} H(t, s) L(s, x(s)) \frac{d s}{s},
\end{aligned}
$$

and the second implies

$$
\begin{aligned}
x(t)-\int_{1}^{t} k_{2}(s) \frac{d s}{s} & =\theta(\log t)^{\alpha-1}+\int_{1}^{e} H(t, s) k_{4}(s) \frac{d s}{s} \\
& \geq \theta(\log t)^{\alpha-1}+\int_{1}^{e} H(t, s) U(s, x(s)) \frac{d s}{s},
\end{aligned}
$$

which are the upper and lower solutions of Equations (3.5), respectively. An application of Theorem 3.1 yields that the FDE (1.1) has at least one solution $x \in X$ and satisfies Equation (3.4).

Corollary 3.2. Assume that (3.2) holds and $0<\sigma<k(t)=\lim _{x \rightarrow \infty} f(t, x)<\infty$ for $t \in[1, e]$. Then the FDE (1.1) has at least a positive solution $x \in X$.

Proof. By assumption, if $x>\rho>0$, then $0 \leq|f(t, x)-k(t)|<\sigma$ for any $t \in[1, e]$. Hence, $0<k(t)-\sigma \leq f(t, x) \leq k(t)+\sigma$ for $t \in[1, e]$ and $\rho<x<+\infty$. Now if $\max \{f(t, x): t \in[1, e], x \leq \rho\} \leq \nu$, then $k(t)-\sigma \leq f(t, x) \leq k(t)+\sigma+\nu$ for $t \in[1, e]$, and $0<x<+\infty$. By Corollary 3.2, the FDE (1.1) has at least one positive solution $x \in X$ satisfying

$$
\begin{aligned}
& \theta(\log t)^{\alpha-1}+\int_{1}^{e} H(t, s) k(s) \frac{d s}{s} \\
& -\frac{\sigma\left((\log t)^{\alpha-1}-(\log t)^{\alpha}\right)}{\Gamma(\alpha+1)}+\int_{1}^{t} k_{1}(s) \frac{d s}{s} \\
& \leq x(t) \\
& \leq \theta(\log t)^{\alpha-1}+\int_{1}^{e} H(t, s) k(s) \frac{d s}{s} \\
& +\frac{(\sigma+\nu)\left((\log t)^{\alpha-1}-(\log t)^{\alpha}\right)}{\Gamma(\alpha+1)}+\int_{1}^{t} k_{2}(s) \frac{d s}{s} .
\end{aligned}
$$

Corollary 3.3. Assume that $0<\sigma<f(t, x(t)) \leq \gamma x(t)+\eta<\infty$ for $t \in[1, e]$, and $\sigma, \eta$ and $\gamma$ are positive constants with $\frac{\Gamma(\alpha) \gamma}{\Gamma(2 \alpha)}<1$. Then, the FDE (1.1) has at least one positive solution $x \in C([1, e])$. 
Proof. Consider the equation

$$
\left\{\begin{array}{l}
\mathfrak{D}_{1}^{\alpha} x(t)+(\gamma x(t)+\eta)=\mathfrak{D}_{1}^{\alpha-1} g(t, x(t)), 1<t<e, \\
x(1)=0, x(e)=\theta+\int_{1}^{e} g(s, x(s)) \frac{d s}{s} .
\end{array}\right.
$$

Equation (3.6) is equivalent to integral equation

$$
x(t)=\theta(\log t)^{\alpha-1}+\int_{1}^{e} H(t, s)(\gamma x(s)+\eta) \frac{d s}{s}+\int_{1}^{t} g(s, x(s)) \frac{d s}{s} .
$$

Let $\omega$ be a positive real number and $\phi=\frac{\Gamma(\alpha) \gamma}{\Gamma(2 \alpha)}<1$ such that

$$
\omega>(1-\phi)^{-1}\left(\theta+\frac{\eta \Gamma(\alpha)}{\Gamma(2 \alpha)}+c_{g}\right) .
$$

Then, the set $B_{\omega}=\{x \in X:|x(t)| \leq \omega, 1 \leq t \leq e\}$ is convex, closed, and bounded subset of $C([1, e])$. The operator $F: B_{\omega} \longrightarrow B_{\omega}$ given by

$$
(F x)(t)=\theta(\log t)^{\alpha-1}+\int_{1}^{e} H(t, s)(\gamma x(s)+\eta) \frac{d s}{s}+\int_{1}^{t} g(s, x(s)) \frac{d s}{s},
$$

is compact as in the proof of Theorem 3.1. Moreover,

$$
|(F x)(t)| \leq \frac{\Gamma(\alpha) \gamma}{\Gamma(2 \alpha)}\|x\|+\theta+\frac{\eta \Gamma(\alpha)}{\Gamma(2 \alpha)}+c_{g}
$$

If $x \in B_{\omega}$, then

$$
|(F x)(t)| \leq \phi \omega+(1-\phi) \omega=\omega
$$

that is $\|F x\| \leq \omega$. Hence, the Schauder fixed theorem ensures that the operator $F$ has at least one fixed point in $B_{\omega}$, and then Equation (3.6) has at least one positive solution $x^{*}(t)$, where $1<t<e$. Therefore, if $t \in[1, e]$ one can asserts that

$$
\begin{aligned}
x^{*}(t) & =\theta(\log t)^{\alpha-1}+\int_{1}^{e} H(t, s)\left(\gamma x^{*}(s)+\eta\right) \frac{d s}{s}+\int_{1}^{t} g\left(s, x^{*}(s)\right) \frac{d s}{s} \\
& =\theta(\log t)^{\alpha-1}+\gamma \int_{1}^{e} H(t, s) x^{*}(s) \frac{d s}{s} \\
& +\frac{\eta\left((\log t)^{\alpha-1}-(\log t)^{\alpha}\right)}{\Gamma(\alpha+1)}+\int_{1}^{t} g\left(s, x^{*}(s)\right) \frac{d s}{s} .
\end{aligned}
$$

The definition of control function implies

$$
\begin{aligned}
& \theta(\log t)^{\alpha-1}+\int_{1}^{e} H(t, s) U\left(s, x^{*}(s)\right) \frac{d s}{s} \\
& \leq \theta(\log t)^{\alpha-1}+\int_{1}^{e} H(t, s)\left(\gamma x^{*}(s)+\eta\right) \frac{d s}{s} \\
& =x^{*}(t)-\int_{1}^{t} g\left(s, x^{*}(s)\right) \frac{d s}{s},
\end{aligned}
$$

then $x^{*}$ is an upper positive solution of the FDE (1.1). Moreover, one can consider

$$
x_{*}(t)=\theta(\log t)^{\alpha-1}+\frac{\sigma\left((\log t)^{\alpha-1}-(\log t)^{\alpha}\right)}{\Gamma(\alpha+1)}+\int_{1}^{t} g\left(s, x_{*}(s)\right) \frac{d s}{s},
$$


as a lower positive solution of Equation (1.1). By Theorem 3.1, the FDE (1.1) has at least one positive solution $x \in C([1, e])$, where $x_{*}(t) \leq x(t) \leq x^{*}(t)$.

The last result is the uniqueness of the positive solution of (1.1) using Banach contraction principle.

Theorem 3.2. Assume that $(H 1)$ and $(H 2)$ are satisfied and

$$
\left(\frac{\Gamma(\alpha) \beta_{2}}{\Gamma(2 \alpha)}+\beta_{1}\right)<1
$$

Then the FDE (1.1) has a unique positive solution $x \in \mathcal{C}$.

Proof. From Theorem 3.1, it follows that the FDE (1.1) has at least one positive solution in $\mathcal{C}$. Hence, we need only to prove that the operator defined in (3.1) is a contraction on $\mathcal{C}$. In fact, for any $x, y \in \mathcal{C}$, we have

$$
\begin{aligned}
& |(\Phi x)(t)-(\Phi y)(t)| \\
& \leq \int_{1}^{e} H(t, s)|f(s, x(s))-f(s, y(s))| \frac{d s}{s} \\
& +\int_{1}^{t}|g(s, x(s))-g(s, y(s))| \frac{d s}{s} \\
& \leq\left(\frac{\Gamma(\alpha) \beta_{2}}{\Gamma(2 \alpha)}+\beta_{1}\right)\|x-y\| .
\end{aligned}
$$

Hence, the operator $\Phi$ is a contraction mapping by (3.7). Therefore, the FDE (1.1) has a unique positive solution $x \in \mathcal{C}$.

Finally, we give an example to illustrate our results.

Example 3.1. We consider the nonlinear Hadamard fractional differential equation

$$
\left\{\begin{array}{l}
\mathfrak{D}_{1}^{\frac{8}{7}} x(t)-\mathfrak{D}_{1}^{\frac{1}{7}} \frac{2+x(t)}{3+x(t)}=\frac{1}{2+t}\left(2+\frac{t x(t)}{3+x(t)}\right), 1<t<e, \\
x(1)=0, x(e)=1+\int_{1}^{e} \frac{2+x(s)}{3+x(s)} \frac{d s}{s}
\end{array}\right.
$$

where $\theta=1, g(t, x)=\frac{2+x}{3+x}$ and $f(t, x)=\frac{1}{2+t}\left(2+\frac{t x}{3+x}\right)$. Since $g$ is nondecreasing on $x$,

$$
\lim _{x \rightarrow \infty} \frac{2+x}{3+x}=\lim _{x \longrightarrow \infty} \frac{1}{2+t}\left(2+\frac{t x}{3+x}\right)=1,
$$

and

$$
\frac{2}{3} \leq g(t, x) \leq 1, \frac{2}{2+e} \leq f(t, x) \leq 1,
$$

for $(t, x) \in[1, e] \times[0,+\infty)$, hence by any of the above Corollaries, the equation (3.8) has a positive solution. Also, we have

$$
\frac{\Gamma(\alpha) \beta_{2}}{\Gamma(2 \alpha)}+\beta_{1} \simeq 0.266<1
$$

then by Theorem 3.2, the equation (3.8) has a unique positive solution. 


\section{References}

[1] B. Ahmad, S. K. Ntouyas, Existence and uniqueness of solutions for CaputoHadamard sequential fractional order neutral functional differential equations, Electronic Journal of Differential Equations 2017(36) (2017), 1-11.

[2] Z. Bai, H. Lü, Positive solutions for boundary value problem of nonlinear fractional differential equation, J. Math. Anal. Appl. 311 (2005), 495-505.

[3] Z. Bai, T. T. Qiu, Existence of positive solution for singular fractional differential equation, Appl. Math. Comput. 215 (2009), 2761-2767.

[4] M. Benchohra, J. E. Lazreg, Existence and Ulam stability for nonlinear implicit fractional differential equations with Hadamard derivative, Stud. Univ. Babes-Bolyai Math. 62(1) (2017), 27-38.

[5] H. Boulares, A. Ardjouni, Y. Laskri, Positive solutions for nonlinear fractional differential equations, Positivity 21 (2017), 1201-1212.

[6] D. Delbosco, L. Rodino, Existence and uniqueness for a nonlinear fractional differential equation, J. Math. Anal. Appl. 204 (1996), 609-625.

[7] E. Kaufmann, E. Mboumi, Positive solutions of a boundary value problem for a nonlinear fractional differential equation, Electron. J. Qual. Theory Differ. Equ. 2008(3) (2008), 1-11.

[8] A. A. Kilbas, H. M. Srivastava, J. J. Trujillo, Theory and applications of fractional differential equations, Elsevier, Amsterdam 2006.

[9] C. Kou, H. Zhou, Y. Yan, Existence of solutions of initial value problems for nonlinear fractional differential equations on the half-axis, Nonlinear Anal. 74 (2011), 5975-5986.

[10] M. Matar, On existence of positive solution for initial value problem of nonlinear fractional differential equations of order $1<\alpha \leq 2$, Acta Math. Univ. Comenianae LXXXIV(1) (2015), 51-57.

[11] K. S. Miller, B. Ross, An introduction to the fractional calculus and fractional differential equations, Wiley, New York, 1993.

[12] I. Podlubny, Fractional differential equations, Academic Press, San Diego, 1999.

[13] D. R. Smart, Fixed point theorems, Cambridge Univ. Press., Cambridge, 1980.

[14] C. Wang, R. Wang, S. Wang, C. Yang, Positive Solution of Singular Boundary Value Problem for a Nonlinear Fractional Differential Equation, Bound. Value Probl. 2011 (2011), Art ID 297026.

[15] C. Wang, H. Zhang, S. Wang, Positive solution of a nonlinear fractional differential equation involving Caputo derivative, Discrete Dynamics in Natural and Society 2012 (2012), Art ID425408.

[16] M. Xu, S. Sun, Positivity for integral boundary value problems of fractional differential equations with two nonlinear terms, J. Appl. Math. Comput., 59(12) (2019), $271-283$

[17] S. Zhang, Existence results of positive solutions to boundary value problem for fractional differential equation, Positivity 13(3) (2009), 583-599.

[18] S. Zhang, The existence of a positive solution for a fractional differential equation, J. Math. Anal. Appl. 252 (2000), 804-812.

Abdelouaheb Ardjouni

Department of Mathematics and Informatics, Faculty of Sciences and Technology, University of Souk Ahras, P.O. Box 1553, Souk Ahras, 41000, Algeria.

E-mail address: abd_ardjouni@yahoo.fr

Received: March 1, 2019; Accepted: May 7, 2019 